\title{
Research on the Application of Information Technology in Marketing and Financial Management
}

\section{Bingtao She}

Chaozhou Branch, Guangdong Co., Ltd. of China Mobile Communications Group, Chaozhou, Guangdong, 521000, China

\begin{abstract}
Traditional business marketing is to separate products and discounts in physical channel stores. Generally, after opening the product in the BOSS system, the front-end manual or back-end batch method is used to handle discounts for customers. This kind of marketing method is costly and inefficient, the customer experience is poor, and the enthusiasm for participating in marketing activities is greatly reduced; it is also difficult for companies to control marketing revenue, and it is prone to exceed budget or revenue failure. This paper proposes to build an intelligent business marketing system of app, WeChat and SMS. The marketing system adopts a distributed architecture to provide customers with the processing channel of app, WeChat and SMS instructions. In the business process design, it realizes the intelligent association of multiple systems, and finally realizes the "one click processing" of products and preferential delivery schemes on mobile phones. After the system is put into use, it greatly improves the business marketing efficiency and company revenue, and realizes the controllability of input resources and enterprise revenue in finance.
\end{abstract}

\section{Keywords}

marketing; intelligence; information technology; financial management

\section{信息技术在市场营销和财务管理中的应用探究}

\author{
余炳涛 \\ 中国移动通信集团广东有限公司潮州分公司，中国・广东 潮州 521000
}

\section{摘 要}

传统的业务营销是在实体渠道店进行产品和优惠分离操作, 一般是先在 BOSS 系统开通产品后通过前台人工或后台批量的方 式为客户办理优惠。这样的营销方式高成本低效率，客户体验较差，参与营销活动的积极性大幅降低; 企业对营销收入的控 制也比较困难，容易出现超预算或收入不达标的情况。论文提出构建 APP、微信和短信的智能化业务营销系统，营销系统采 用分布式架构, 为客户提供 APP、微信和短信指令的办理通道, 在业务流程设计上实现多系统智能化关联, 最终实现产品和 优惠赠送方案在手机上 “一键办理”。系统投入使用后，大幅提升业务营销效率和公司收入，并在财务上实现了对投入资源 和企业收入可控性。

\section{关键词}

市场营销; 智能化; 信息技术; 财务管理

\section{1 市场营销和业务支撑中存在的问题}

\section{1 营销渠道覆盖不足}

目前，在中国移动的业务销售渠道中，营业厅和社会渠 道点服务是面向客户的最直接手段, 然而单单营业厅和社会 渠道点服务不能够完全满足整个客户群服务需求，还有广阔 的农村市场和边远山区个人客户未能覆盖。在营销渠道中， 不同分类的客户需提供差异化的特定服务，而这恰恰对营业 网点的覆盖率提出较大的挑战。

【作者简介】余炳涛（1979-）, 男, 中国广东潮州人, 研 究生学历, 高级经济师, 从事工商管理研究。

\section{2 业务支撑效率低下}

当前大多数渠道的主要销售方式仍为线下人员 “一对一” 模式，一般是客户在前台办理产品之后再办理优惠，或者再 由后台批量开通优惠，但无论是通过前台单独办理或者后台 批量办理, 均需要经过种种校验（如方案互斥检验、目标客 户校验）等数据处理后才能办理。对于前台人员，校验是通 过手工进行的, 如果活动优惠幅度较大, 前台容易排起长队, 如果客户不满足其中一个条件，还要先为客户处理其它情况 后再办理，从而降低营销效率。对于后台支撑人员，要进行 业务批量办理需要经过 “多次提数、数据匹配、多次领导审批、 
批开前短信告知” 等环节，才能最终实现业务办理。在激烈 的电信市场竞争环境下, 整个业务支撑的流程就显得费时费 力, 效率十分低下。

\section{3 客户体验较差、业务收入可控性差}

近些年来, 中国电信市场发展突飞猛进, 在此过程中为 应对产业竞争往往过分关注销售业绩, 而忽视了用户体验这 个重要环节。如用户在办理许多业务时仍旧需要前往就近网 点进行咨询办理, 过程繁琐且耗时耗力, 拖沓的业务办理环 节不可避免影响了用户的使用感知; 其次, 一些对价格不敏 感的客户会放弃优惠, 从而降低营销的效果; 而且传统的优 惠营销都是先开业务, 然后通过后台 BOSS 批量增送优惠, 客户感知差, 赠费到账至少要 $30 \mathrm{~d}$ 以上, 有的甚至 $60 \mathrm{~d}$, 效率低, 容易引起客户投诉。

另一方面, 当参加人数暴增, 业务的赠送优惠较大时, 投入资源会超出预算, 容易引起公司减收; 而当参与人数不 足时, 业务办理较少, 企业收入指标会完成不了。这样对企 业管理非常不利。

\section{2 存在问题分析与对策}

首先, 客户对服务的要求越来越高, 要求渠道能提供及 时、便利、不间断的服务, 给渠道的响应提出了更高的标准 要求。这就需要不断加大对渠道网点的建设力度, 力求完善 服务区域的渠道覆盖率。但随着人群的流动性及不确定性, 实体渠道的建设往往较为被动, 不能第一时间满足线下人群 的服务需求。随着移动互联网的普及, 手机应用已经被广泛 应用, 微信、短信、APP 已经成为人们常用的终端应用服务。 假如将应用和短信的做成一个业务办理渠道, 则具有广泛的 客户基础, 又能解决渠道的覆盖问题。

其次, 客户的体验式消费意识不断增强, 要求渠道提供 交互式、体验式的线上服务，线下 “一对一” 的服务模式已 经过时, 产品和优惠分离办理的人工或者半人工的做法已经 不能满足 $4 \mathrm{G}$ 时代的竞争需要, 高效的服务机制和良好的客 户体验将成为赢得市场竞争的关键。假如运用信息化手段打 通各业务办理系统的关联, 实现 “产品优惠一键式” 办理, 且所有的办理情况通过手机终端直接给与客户反馈, 则可以 将前台服务和后台支撑的工作整合为全自动化线上办理, 在 提升客户体验的同时也提升营销效率。当所有业务通过线上 办理，企业收入和资源投入情况基本可以实现实时统计，则
可以在财务上做到可控。

因此，论文提出构建基于手机终端的信息化营销平台的 解决方案, 实现客户在手机上通过短信或手机应用软件进行 产品和优惠的办理。

\section{3 基于手机终端的信息化营销平台 \\ 3.1 营销平台的模块化架构}

信息化营销平台的后台系统由以下各模块组成: (1)业务 引擎模块: 负责处理活动核心流程; (2)分发引擎模块: 负责 将短信上行、微信或和商汇 APP 的参与活动的用户分发到各 个业务引擎处理; (3)后台管理模块: 配置活动、查看活动办 理情况、短信日志等; (4)定时任务模块: 定时调度处理, 如 每天定时上传当天活动办理情况到指定服务器; (5)多渠道调 用模块：提供接口，接受短信、手机应用、微信等渠道的客 户参与活动的申请; (6)短信组件模块: 与短信营业厅交互, 获取通过短信上行的参与活动的用户; 下发短信; (7) ECOP 接口模块: 系统通过 webservice 接口与 ecop 能力平台连接, 再通过 ecop 接口实现与 boss 的交互, 实现方案查询、方案办理、 余额查询、激活日期查询等一系列功能; (8)缓存服务: 用于 存放活动指令以及活动目标号码, 提高活动流程的处理速度。

\section{2 营销平台性能指标设计}

通过并发压力测情况来看, 各线上渠道的上行并发能力 每秒可以达到 300 400 之间, 且服务器不会出现异常情况。 当一个活动总共调用 4 次 BOSS 接口, 那么该活动的处理量 一小时可达到 12000 条左右; 当一个活动是单纯登记 (无任 何目标号码、无需调用 BOSS, 只登记号码) , 单个业务引 擎一分钟可以登记 360 条, 一小时可以登记 21600 条 ${ }^{[1]}$ 。

\section{3 主要业务功能}

\subsection{1 数据业务查询}

(1)提供业务活动办理列表的查询、导出 EXCEL 功能, 点击详细可以查询用户办理过程中的办理明细。(2)办理列表 的信息包括: 手机号码、业务活动编码、业务活动名称、办 理状态 (办理中、成功、失败、取消)、节点状态、办理时间、 完成时间、取消时间、办理渠道、办理工号 (如果是短信办 理则显示系统工号)、接人方式、备注。(3)办理明细的信息包 括活动的信息、用户的信息，用户目前所处的流程的节点， 以及用户办理业务活动的交互明细, 交互明细信息包括: 节 点、上行指令、节点状态、上行时间、下行内容、下行时间、 
下行状态。

\subsection{2 短信日志查询}

为方便进行业务分析 / 故障处理等工作, 平台提供短信 上行下行日志查询功能。具体包括: 按手机号码、上行时间 范围、上行端口、下行端口查询短信日志; 短信日志的信息 包括：手机号码、上行端口、上行时间、短信内容、下行端 口、下行内容、下行时间、递送状态、下行内容、下行时间、 递送状态、下行内容、下行时间、递送状态 ${ }^{[2]}$ 。

\section{3 .3 数据配置管理}

为更明确调用接口的调用者信息及调用的安全性, 为每 个需要调用接口的 IP 授权, 并记录调用着的信息, 调用接口 的类型。具体包括：(1)提供端口的创建功能，接口配置信息包 括：接口调用标识，接口调用人员，授权 ip，调用接口类型， 调用频率；2提供接口配置的编辑功能；(3)提供接口配置的删 除功能, 支持批量删除; (4)提供接口配置的查询功能; (5)提供 多渠道接口让外围系统（微信, 和商汇, app ) 调用短厅活动。

\subsection{4 号码群管理}

为支撑业务活动办理, 系统提供号码群 (目标号码和非 目标号码）管理功能, 包括号码群的创建、编辑、删除、查询、 批量导入导出, 有部分号码群需要每天新增号码。如果群已 经失效, 在业务活动的目标号码中不再做判断。在群信息中 可以查看到该群是否被有效活动所使用 ${ }^{[3]}$ 。具体包括: (1)提 供目标号码的创建功能, 号码群信息包括用户移动号码, 号 码群的信息包括：群名称、生效时间、失效时间、创建时间、 创建人、最后更新人、最后更新时间。点击可以查看详细的 目标号码, 在创建的时候可以不导入号码; 2)提供号码群的 编辑功能, 提供号码的单个和批量删除, 导入时在现有目标 号码的基础上新增, 如果该号码群属于事件营销类, 将对新 推送过来的号码以短信方式进行业务的推广; (3)提供号码群 的查询功能, 按号码群名称模糊查询。

\section{4 信息化营销平台的业务流程仿真设计和营销 应用}

\section{1 业务流程设计}

除了购机优惠和预存优惠外, 大部分的营销活动均可以 在信息化营销平台进行加载营销。要实现在信息化营销平台 上加载, 市场部支撑人员需要根据产品经理提供的的活动方 案利用 VISIO 软件设计业务流程, 并为每个流程配置一个短
信指令, 再送程序员进行流程开发、测试。业务办理完全仿 造 BOSS 系统的功能且可以实现与其他业务流程的关联, 最 终实现的效果与人工操作完全相同。

每个营销活动的业务流程最终以 JAVA 代码的形式在 系统的业务引擎中运行, 可以实现以下主要自动化功能: (1 目标客户判断：通过将营销目标号码事先加载进短厅数据 库, 可实现业务内容的精准推广; 2)重复办理判断：在调用 BOSS 接口的前提下, 能实现方案办理情况的判断, 避免重 复办理; (3)是否充值：通过接口能检测到短信上行号码的余 额变动情况, 如流入、流出, 以及精确到具体的充值金额; (4)业务办理校验: 针对一些特殊营销案例在办理过程中出现 的冲突情况, 能事先校验是否办理通过, 避免后续的流程冗余; (5)激活时间判断: 针对一些需判断激活时间节点的营销活动, 能精准判断用户的激活时间; 6低值养卡黑名单判断：如客 户为低值养卡黑名单用户，则提前下发引导短信; (7)特殊方 案判断：在设置营销活动之前可先将一些已办理的方案进行 判断, 如已办理则下发短信结束流程; 8方案办理成功判断: 通过短厅加载为用户办理的业务, 能根据接口返回的报文判 断是否已办理成功; (9)是否已上行过相同指令: 针对一些业 务如不能重复办理, 需限制用户的办理次数; 10办理失败挂 起：如系统问题等，能通过灵活设置挂起时间，在特定的时 间节点继续为用户办理方案 ${ }^{[4]}$ 。

\section{2 流量套餐优惠活动的应用案例}

为进一步培养用户流量使用习惯, 激发流量使用需求, 结合 2017 年中国潮州公司收入考核指标要求, 潮州公司在 2017 年 4 月至 11 月持续开展 360 元流量套餐优惠活动。在 充分考虑营销渠道的自动化功能以及流量套餐业务特点的情 况下，开发设计出 LLNB 业务流程。LLNB 业务流程经程序 员转化成 JAVA 代码后放在营销系统的业务引擎运行, 实现 了在业务营销系统上加载 360 元流量套餐产品及赠费优惠。 在业务流程正式上线对外开放时, 潮州移动客户可以通过短 信、潮州移动微信公众号、手机应用发送一个指令 LLNB 即 可完成产品和优惠的自动化办理。

\section{5 在营销实践中的应用成效}

\section{1 整体应用成效}

系统自 2015 年底投入使用, 累计开发的不同业务流程 $($ 业 务指令）超过 50 个, 每年客户上行量超过 200 万，业务办理 
量超过 100 万, 为公司创收超过 1.5 亿元。业务实现在手机 端线上办理后，为公司业务支持中心和市场部大量节省了后 台业务支撑的工作量并降低了人工提数的出错率，同时大幅 节省了县区分公司人力物力并提升了营销效率。

\section{2 创造单日业务办理超过 6 万户的历史最高纪录}

2016 年 5 月, 潮州移动开展 “517 电信日充值 50 元送 50 元优惠活动” , 针对以往在电信日出现大量客户在前台排长队 办理的问题, 本次活动首次启用智能化业务营销系统进行业务 办理, 客户通过空中充值、微信、前台等进行充值后发送短信 CZWS 到 1008611, 实现单日业务成功办理量超过 6 万户的历 史最高纪录, 比营业厅前台人工办理量 (每日 6000 户) 提升了 10 倍, 实现零排队; 每个客户充值 50 元, 则单日创收 300 万元。

\section{3 流量年包优惠单个活动创收近 1 亿元}

结合 2017 年潮州公司收人考核指标要求, 潮州公司在 2017 年 4 月至 11 月, 持续开展 360 元流量年包优惠活动, 累计参与业务量 53 万次, 项目成功办理用户 28 万户，每户 产生收入 360 元 (每户每月 30 元 *12 个月 ), 日均成功办理 1300 户，合计创造收入近 1 亿元

\section{6 实现创新点}

\section{1 产品和优惠一键化办理信息化}

运用信息化手段实现产品和优惠的远程同步办理, 让客 户 “足不出户, 产品和优惠一键办理” , 为公司渠道体系增 添了一个信息化渠道对提升了提升公司服务和业务收入具有 重大意义。

\section{2 在市场营销中实现人机交互人性化}

平台能通过短信下发或业务弹窗的方式对问题进行提示 和引导, 特别是及时说明业务办理不成功的原因和解决办法。 在提升用户使用感知的同时，很大程度上缓解了营业前台的 工作积压问题，减少前台人员工作压力。

\section{3 企业财务收入可调性}

营销平台可实时监控客户的办理情况，整个营销活动的 资源投入和收入情况可以实时统计。当收入指标完成时, 可 以将用户的需求指令自动延后到下个月或以后某个时间再办 理, 即把客户需求挂起后通过短信告知和解释, 等于是把收 人调节到以后月份; 当收入指标未完成时, 立即启动 10086 外呼业务推广, 将客户数据送至 FTP 平台, 营销平台定时下 载这个 FTP 的数据, 通过批量办理的方式为客户自动开通业
务以提升收人。

\section{7 应用领域展望与意义}

第一, 系统接口模块是独立可扩展的, 有较强的可扩展 性和兼容性; 如果未来有其它热门手机应用或应用系统开发 出来, 我们系统可以通过扩展系统接口模块进行整合; 比如, 当前广东移动 $10086 \mathrm{APP}$ 访问量很大, 未来系统升级可以将 广东 10086APP 整合进来。第二, 购机业务是我们重点业务, 但目前仍不能在系统上实现办理; 如果系统能打通微信支付 与 BOSS 系统对接, 加上物流配送环节的辅助, “足不户, 一键购机” 完全可以实现。第三，论文所提到的微信和和商 汇仅指是潮州本地 “潮州移动微信公众号” 和 “潮州和商汇; 在全省集中化 IT 支撑的背景下, 此次业务营销系统的成功研 发和应用, 对于研究和开发基于 “广东移动微信公众号”、“广 东移动和商汇” 的全省性智能化业务营销平台具有重大借鉴 意义。省公司可以在接口扩展、容量拓展和地市分区方面做 进一步的研究和探索。第四, 由于 BOSS 接口调用比较费时, 单个业务流程中调用 BOSS 的次数越多, 则单位时间内的业 务办理数量会下降, 因此在开展重大节日营销前, 要先根据 业务流程的复杂程度充分评估系统的处理能力, 并以此决定 系统开发中业务引擎的数量。

\section{8 结语}

论文针对当前市场营销中存在的问题进行了深入的分 析, 提出了建设基于手机终端的信息化营销平台的解决方案, 重点阐述了平台构建、业务流程设计以及在营销实践所带来 的效益。经营销实践证明，该系统的成功研发和应用，丰富 了公司渠道体系, 降低营销成本, 提升客户感知和公司收入。 后续将根据运行的情况，开展针对性系统优化，不断契合当 下市场营销需求，为公司业务版图的扩展 “保驾护航”。

\section{参考文献}

[1] 杨等立. 电信营销渠道现存问题及原因分析 [J]. 信息通信, 2013(04):235-236.

[2] 黄帝媛, 微信营销的现状及发展趋势 [J]. 智富时代, 2016(07):55-56.

[3] 夏铭翊. 新经济背景下企业市场营销战略分析 [J]. 经营管理 者, 2011(18):170-170.

[4] 汤利颖. 电信企业客户服务质量感知与客户满意度研究 [J]. 统计 与决策 , 2010(08):100-102. 